\title{
The new indexes comparing the radicality of tumor removal and the extent of post-operative defects after treatment of basal cell cancer by mean of Mohs Surgery and Classical Excision
}

\author{
Andrzej Bieniek ${ }^{1,2 *}$, Łukasz Matusiak ${ }^{1}, Z_{\text {dzisław Woźniak }}{ }^{1}$, Karolona Wójcicka ${ }^{1}$ and Maria Kozioł ${ }^{1}$ \\ ${ }^{1}$ Department of Dermatology, Venereology and Allergology at Wroclaw Medical University, Poland \\ ${ }^{2}$ Private Center for Plastic and Skin Surgery - Andrzej Bieniek, Wrocław, Poland
}

\begin{abstract}
Mohs Surgery (MMS) and Excision with Predetermined Margins (EPM) vary in form and width of tumor removal. Exact measurements and calculations of the related values enable to compare precisely those methods.

Materials and methods: In 668 BCC cases treated with MMS, the following measurements and calculations were carried out: a) the widest margin of excision [Marg max MMS], b) the mean margin of excision [Marg mean MMS], c) the hypothetical margin of excision in EPM [Marg EPM]. The two comparative indexes were proposed: d) index of radicality of tumor removal [IRTR] - calculated by dividing [Marg max MMS], and [Marg EPM], and e) index of extensions of the defects [IED] - calculated by dividing the [Marg mean MMS] and [Marg EPM].
\end{abstract}

Results: a) [Marg max MMS] ranged from $1 \mathrm{~mm}$ to $40 \mathrm{~mm}$, b) [Marg mean MMS] - 1 to $24.1 \mathrm{~mm}, \mathrm{c}$ ) [Marg EPM] - $1 \mathrm{~mm}$ to $15 \mathrm{~mm}$, d) [IRTR] ranged 33,3\% to $444 \%$ (on average $106.20 \%$ ), e) [IED] - 33,3\% to $344.41 \%$ (on average $84.93 \%$ ).

Conclusions: The excision of BCC with MMS was performed more radically (by mean of $6.2 \%$ - not singificant statistically), the extent of defects was smaller (by mean of $15.07 \%$-statistically significanct) - compared to EPM.

\section{Introduction}

Excision by Mohs Micrographic Surgery (MMS) or excision with predetermined margins (EPM) belong to the most common treatment methods of Basal Cell Cancers [1]. The basic dilemma for surgeons who uses the procedure of EPM is the necessity of choosing the most appropriate width of margins. On the basis of common recommendations, lateral margins of classical excision should be equal in all directions, whereas their width should be differentiated, depending on individual features of tumor aggressiveness. However, such recommendations are not straightforward [2]. Besides, to assure high probability of eradication of BCC by EPM (as well as high cure rate), especially in "high-risk" tumors, it is imperative to employ relatively wide excision margins [3-5]. Despite this attitude, the cure rates in this group seem not to be optimal [6]. It may also lead to unnecessary sacrifice of healthy tissues, what becomes particularly undesirable in anatomical areas most important from the aesthetic and functional point of view. Recommendations of deep margins of excision are much more explicit; in less complicated cases the excision depth at subcutaneous layer is commonly accepted.

The Mohs Micrographic Surgery (MMS) allows to overcome the difficulties with outlining appropriate margins of excision. Depending on actual range of malignant infiltrations it clearly defines the area of excision, enabling both the radical and selective tumor removal [6-8] (Figures 1 and 2).
The MMS technique has been practiced at the Unit of Plastic Surgery, Department of Dermatology, Venereology and Allergology, Medical University of Wroclaw since 1994, lately in Private Center for Plastic and Skin Surgery. At that time 930 tumors have been treated, above all - high risk BCC's, as well facial tumors located in areas most substantial for aesthetic and functional reasons. That constituted in different years from 10 to $20 \%$ of all cases of the tumors treated (more often excised "classically" with use of EPS). Among 668 cases of BCC treated between 1994 and 2010-414 cases (61.97\%) were primary, 231 (34.58\%) - recurrent (Figure 2) and 23 cases (3.44\%)-represented cases of earlier non-radical excisions (excisio non in sano). Size of treated tumors (measured along the greater diameter) ranged from 2 to 150 $\mathrm{mm}$, on average $19.014 \mathrm{~mm}$, median 15.00, standard deviation SD of 16.362. BCC of "high risk histologic types" (infiltrative, morpheiform, micronodular, metatypic) were recognized in 398 cases $(59.58 \%)$,

Correspondence to: Andrzej Bieniek, Department of Dermatology, Venereology and Allergology at Wroclaw Medical University, Poland, E-mail: abieniek@cmbieniek.pl

Key words: basal cell cancer, Mohs micrographic surgery, excision with predetermined margins, width of excision margins, extent of post-operative defects, comparison of treatment methods

Received: January 24, 2017; Accepted: February 09, 2017; Published: February 13,2017 


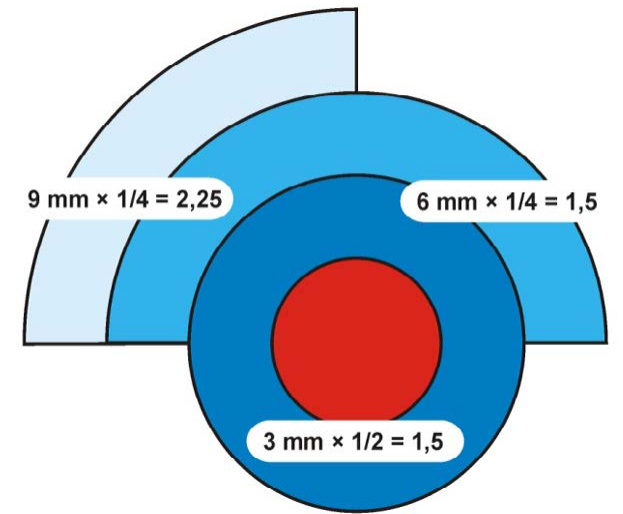

Figure 1. The treatment scheme by MMS technique in which the excision of the margins of $3 \mathrm{~mm}$ was done threefold on various parts of the periphery $(100,50,25 \%)$. The narrowes lateral margin of excision (Marg min MMS) $=3 \mathrm{~mm}$, the widest lateral margin (Marg max MMS $)=9 \mathrm{~mm}$, whereas the mean lateral margin $($ Marg mean MMS $)=5.25 \mathrm{~mm}[(3 \times 50 \%)$ $+(6 \times 25 \%)+(9 \times 25 \%)]$ or $[(3 \times 100 \%)+(3 \times 50 \%)+(3 \times 25 \%)]$.

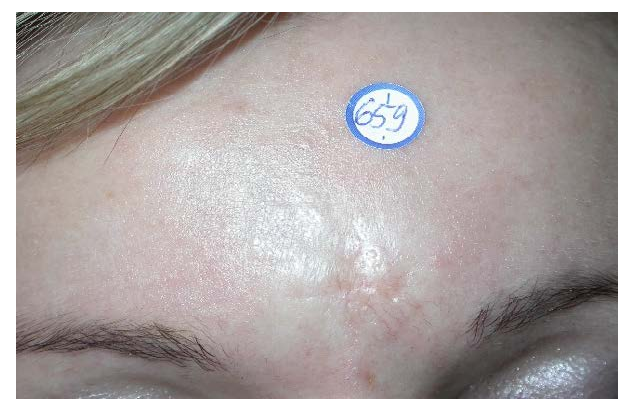

Figure 2A. Recurrent, morphea-like Basal Cell Cancer (fourth relapse after treatment with photodynamic therapy and cryosurgery), with unclear borders, measuring $22 \times 25 \mathrm{~mm}$. Width of hypothetical standard excision margin set at $6.5 \mathrm{~mm}$ (arithmetic mean of the largest hypothetical $(8 \mathrm{~mm})$ and smallest $(5 \mathrm{~mm})$.

"low risk types" (solid, adenoid, cystic, superficial, pigmented) - in 232 cases $(34.73 \%)$, whereas in 38 cases $(5.68 \%)$ the histologic pattern was unidentified. The excision width at each stage ranged from 1 to 5 $\mathrm{mm}$ (on average $2.23 \mathrm{~mm}$ ), depending on the probability of malignant infiltration and tumor location (the narrowest margins were employed in the orbital area).

To our knowledge, no medical publication which would enable to determine the exact relations between excision margins in MMS and EPM (considered by majority of surgeons worldwide to be basic) has been presented so far. Therefore the authors set their sights on measuring and elaborating the comparative indexes, describing in particular the rate of radicality (completeness) of tumor removal, as well as the rate of extension of post-operative defects.

\section{Materials and methods}

In every of 668 cases of Basal Cell Cancer (BCC) treated by Mohs Micrographic Surgery (MMS) between 1994 and 2010 the following values were defined:

a) Maximum width of excision in MMS [Marg max MMS] (measured at the last stage of proceedings from the noticeable border of a tumor to the furthest excision margin) (Figures 1 and $2 \mathrm{~B}$ ). The value may be regarded as equivalent of the necessary width of Excision with Predetermined Margins (EPM), indispensable for complete tumor eradication. It also reflects approximately the extensiveness of tumor infiltration. b) Mean width of excision in MMS [Marg mean MMS] (calculated as sum of the products of given width of excision margin's and the corresponding fraction of excision perimeter. (Figures 1 and 2B). This value reflects approximately the extent of post-operative defect.

c) Hypothetical width of Excision with Predetermined Margins [Marg EPM]. It was defined by arithmetic mean between the maximum hypothetical margin (designed with intention of full, unquestionable cure) and the "sparing" hypothetical margin (designed with intention of tissue conservation and sufficient probability of cure). The value of [Marg EPM] expressed both, the quality of rationally high curability and at the same time-certain tendency for preservation of tissue. (Figures 1 and 2B)

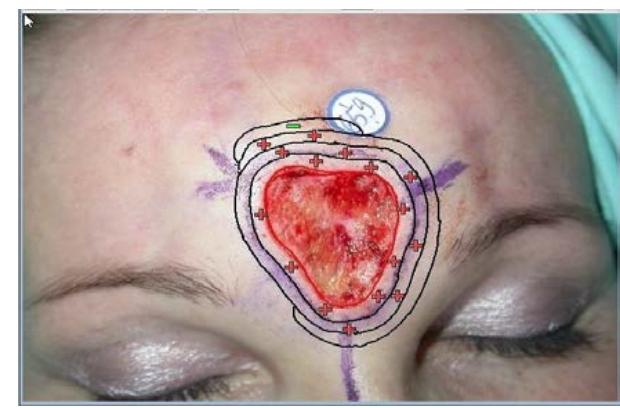

Figure 2B. Malignant infiltrates and the stages of their serial excisions are illustrated on digital map of the tumor. The treatment method by Mohs Micrographic Surgery was carried out in four stages, performing the resection at each of the stages at various parts of the periphery tissues' margin of $3 \mathrm{~mm}$. Minimum excision margin in MMS technique was in this case $6 \mathrm{~mm}$, the maximum excision margin- $12 \mathrm{~mm}$, the average excision margin $8.7 \mathrm{~mm}$ $(3 \times 100 \%+3 \times 100 \%+3 \times 75 \%+3 \times 15 \%)$

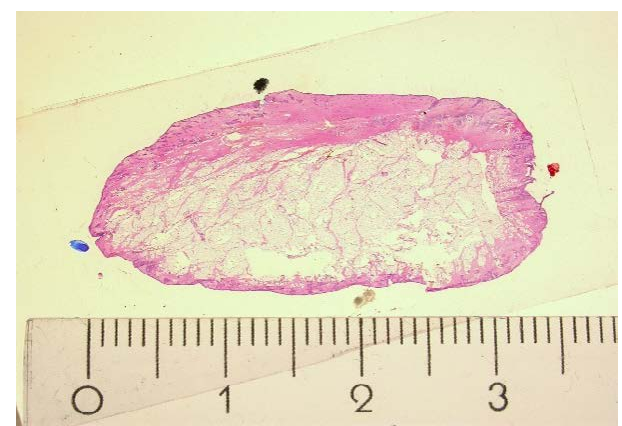

Figure 2C. The own technique of histological processing allows to perform large tissue sections. In this case, one histologic specimen represents tissues of $34 \times 37 \mathrm{~mm}$ (the dimensions of histologic speciemen are about $10-20 \%$ smaller than raw tissue due to the shrinkage during preparation).

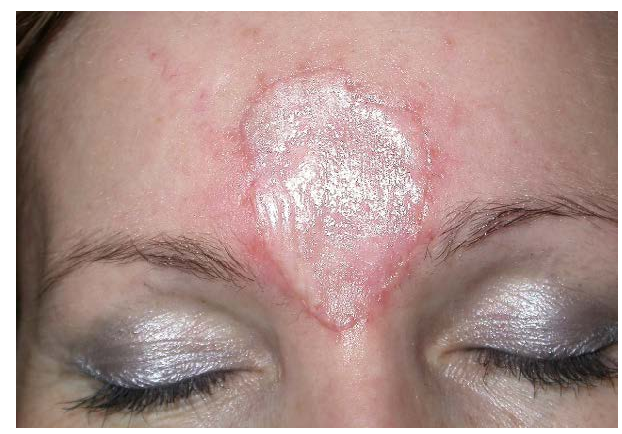

Figure 2D. The final lesions, with unpredictable shapes and sizes was covered with a fullthickness skin graft. The index of completess of tumor removal (ICR) was $184 \%$ (12:6.5), and the index of extensions of the defect (IED) was $133 \%(8,7: 6.5)$. In demonstrated case, the technique of MMS proved to be more radical (of $84 \%$ ), and led to the more extensive tissue lesion (of 33\%), compared to a hypothetical standard Excision with Predetermined Margins. 
Bieniek A (2017) The new indexes comparing the radicality of tumor removal and the extent of post-operative defects after treatment of basal cell cancer by mean of Mohs Surgery and Classical Excision

The values of [Marg max MMS] and [Marg mean MMS] were compared (by division) to [Marg EPM], thus determining the two new indexes:

1. The index of radicality of tumor removal [IRTR] [Marg max MMS] / [Marg EPM].

2. The index of extent of postoperative defects [IED] [Marg mean MMS] / [Marg EPM].

The data were collected, systemized and pre-analyzed using the Excel 2010 tools. The same spreadsheet was also used to calculate the mean values of the studied parameters, variation and incidence of certain states and graphs. For further analysis, the package Statistica 10.0 GB was used. For all quantitative study statistically significant level of $\mathrm{p}<0.05$ was considered. With the rejection of the hypothesis of the normality of distribution of studied variables by Shapiro-Wilk test, the non-parametric Wilcoxon matched pairs test was used in comparisons.

\section{Results}

a) [Marg max MMS] ranged from $1 \mathrm{~mm}$ to $40 \mathrm{~mm}$ (on average 5,07 mm, median 4,00, SD 3,79) (Table 1, Figure 3).

Table 1. Margins of excisions of BCC's in Mohs Micrographic Surgery - the largest [Marg max MMS] and mean [Marg mean MMS], as well as the hypothetical margins of standard Excision with Predetermined Margins [Marg EPM], calculated during the treatment of 668 cases of Basal Cell Cancers, in increments of $5 \mathrm{~mm}$.

\begin{tabular}{|l|c|c|c|c|c|c|}
\hline & \multicolumn{2}{|c|}{ [Marg.max.MMS] } & \multicolumn{2}{c|}{ [Marg.mean.MMS] } & \multicolumn{2}{c|}{ [Marg.EPM] } \\
\hline $\begin{array}{l}\text { Width of excision } \\
\text { margins }(\mathrm{mm})\end{array}$ & $\mathrm{N}$ & $\%$ & $\mathrm{~N}$ & $\%$ & $\mathrm{~N}$ & $\%$ \\
\hline$(0 ; 5]$ & 466 & $69.97 \%$ & 569.00 & $85.44 \%$ & 553 & $83.03 \%$ \\
\hline$(5 ; 10]$ & 172 & $25.83 \%$ & 85.00 & $12.76 \%$ & 110 & $16.52 \%$ \\
\hline$(10 ; 15]$ & 16 & $2.40 \%$ & 9.00 & $1.35 \%$ & 3 & $0.45 \%$ \\
\hline$(15 ; 20]$ & 6 & $0.90 \%$ & 1.00 & $0.15 \%$ & 0 & $0.00 \%$ \\
\hline$(20 ; 25]$ & 1 & $0.15 \%$ & 2.00 & $0.30 \%$ & 0 & $0.00 \%$ \\
\hline$(25 ; 30]$ & 3 & $0.45 \%$ & 0.00 & $0.00 \%$ & 0 & $0.00 \%$ \\
\hline$(30 ; 35]$ & 0 & $0.00 \%$ & 0.00 & $0.00 \%$ & 0 & $0.00 \%$ \\
\hline$(35 ; 40]$ & 2 & $0.30 \%$ & 0.00 & $0.00 \%$ & 0 & $0.00 \%$ \\
\hline
\end{tabular}

b) [Marg mean MMS] ranged from $1 \mathrm{~mm}$ to $24.1 \mathrm{~mm}$ (on average $4,01 \mathrm{~mm}$, median 3,44 mm, SD 2,24). (Table 1 and Figure 3).

c) $[$ Marg EPM] was in each case from $1 \mathrm{~mm}$ to $15 \mathrm{~mm}$ (on average 4,62 mm, median 4,5, SD 0,54). (Table 1 and Figure 3).

d) [IRTR] ranged from $33,33 \%$ to $444 \%$ (on average $106,20 \%$, median 91,61\%, SD 0,54). (Table 2 and Figure 4). The difference between [Marg max MMS] and [Marg EPM] was not statistically singificant $(\mathrm{p}=0,157)$

e) [IED] ranged from $33,33 \%$ to $344.41 \%$ (on average $84,93 \%$, median $80,00 \%$, SD 0,27) (Table 2 and Figure 4). The difference between [Marg mean MMS] and [Marg EPM] was statistically singificant $(\mathrm{p}=0,000)$

\section{Discussion}

The largest lateral excision margin of BCC's in MMS [Marg max MMS] (corresponding approximately to the range of malignant infiltration as well as the radicality of tumor excision) ranged from 1 to $40 \mathrm{~mm}$ (on average $5.07 \mathrm{~mm}$, median 4.00, SD 3.79) (Figure 3). In a significant proportion of cases these margins were extensive; in $30.03 \%$ of cases they exceeded $5 \mathrm{~mm}$; in $4.20 \%-10 \mathrm{~mm}$; and in $1.80 \%-15$ $\mathrm{mm}$. (Table 1, Figure 2, Figure 3 ). In rare cases they reached nearly 4 $\mathrm{cm}$ from the visible boundary of the tumor. Such extensive malignant infiltration was not in any way possible to predict before the surgery. Our results are consistent with the data presented by Leibovitch who found extensive infiltrations of BCC's in approximately $20 \%$ of cases. The results of our investigations confirmed, that the average aggressiveness of tumors in our patients was high. We also revealed statistically significant differences between the range of lateral malignant infiltration of primary BCC's $(4.88 \mathrm{~mm})$ and secondary BCC's $(6.27 \mathrm{~mm})(\mathrm{p}=0.012947)$, groups of BCC's with high $(6.17 \mathrm{~mm})$ and low $(4.04 \mathrm{~mm})$ histological recurrence risk $(\mathrm{p}=0.000,000)$, and the groups of tumors of various sizes $<5 \mathrm{~mm}(3.33 \mathrm{~mm}), 5 \leq \mathrm{x}<10(4,12$ $\mathrm{mm}), 10 \leq \mathrm{x}<20(5.03 \mathrm{~mm}), 20 \leq \mathrm{x}<50(6.53 \mathrm{~mm}), 50 \leq \mathrm{x}(9.68 \mathrm{~mm})$ $(\mathrm{p}=0.000000-0,025933)$. Similar differences were claimed by other researchers [9].

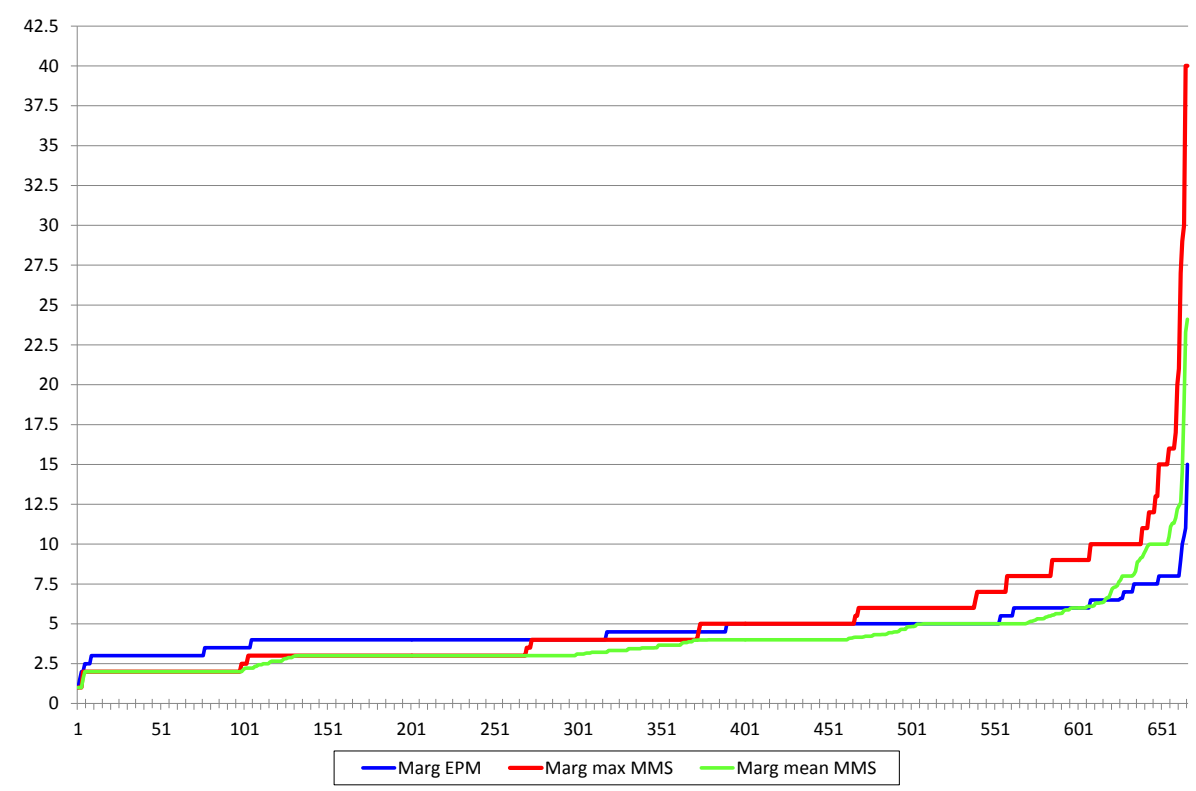

Figure 3. The largest margin of excision in MMS [Marg max MMS] (red line), the mean margin of excision in MMS [Marg mean MMS] (green line), and the hypothetical width of standard excision with predetermined margins [Marg EPM] (blue line), determined during treatment of 668 Basal Cell Cancers with use of Mohs Micrographic Surgery (X-axis - depicts number of patients, Y-axis depicts width of given margins in millimeters). 
Bieniek A (2017) The new indexes comparing the radicality of tumor removal and the extent of post-operative defects after treatment of basal cell cancer by mean of Mohs Surgery and Classical Excision

The mean width of the lateral excision margins of BCC in MMS [Marg mean MMS], corresponding to the extent of postoperative defects ranged from 1 to $24.11 \mathrm{~mm}$, mean $-4.01 \mathrm{~mm}$, median 3,44 , SD 2,24 (Figure 3). It was lower by $20.91 \%$ from the average width of the largest excision margin [Marg max MMS]. This reflected the independence of the two attributes of the MMS - radical excision and tissue preservation.

Hypothetical width of standard Excision with Predetermined Margin [Marg EPM] was determined in each case individually, in relation to the known factors of the aggressiveness of tumors. Its value ranged in each case from $1 \mathrm{~mm}$ to $15 \mathrm{~mm}$ (on average $4,62 \mathrm{~mm}$, median 4,5, SD 1,27). (Figure 3). A much smaller scatter of its values (lower standard deviation) demonstrate that the predictable extent of

Table 2. Indexes of radicality of tumor tumor removal [IRTR] and of the extent of the postoperative defects [IED] -calculated during the treatment of 668 Basal Cell Cancers byMohs Micrographic Surgery method, in increments of values of 0,25

\begin{tabular}{|l|c|c|c|c|}
\hline Value of indexes & \multicolumn{2}{|c|}{ IRTR index } & \multicolumn{2}{c|}{ IED index } \\
\hline & $\mathrm{N}$ & $\%$ & $\mathrm{~N}$ & $\%$ \\
\hline $0 ; 0.25]$ & 0 & $0.00 \%$ & 0 & $0.00 \%$ \\
\hline$(0.25 ; 0.5]$ & 54 & $8.11 \%$ & 57 & $8.56 \%$ \\
\hline$(0.5 ; 0.75]$ & 196 & $29.43 \%$ & 241 & $36.19 \%$ \\
\hline$(0.75 ; 1]$ & 180 & $27.03 \%$ & 255 & $38.29 \%$ \\
\hline$(1 ; 1.25]$ & 72 & $10.81 \%$ & 71 & $10.66 \%$ \\
\hline$(1.25 ; 1.5]$ & 69 & $10.36 \%$ & 26 & $3.90 \%$ \\
\hline$(1.5 ; 1.75]$ & 33 & $4.95 \%$ & 7 & $1.05 \%$ \\
\hline$(1.75 ; 2]$ & 32 & $4.80 \%$ & 8 & $1.20 \%$ \\
\hline$(2 ; 2.25]$ & 8 & $1.20 \%$ & 0 & $0.00 \%$ \\
\hline$(2.25 ; 2.5]$ & 9 & $1.35 \%$ & 0 & $0.00 \%$ \\
\hline$(2.5 ; 2.75]$ & 1 & $0.15 \%$ & 0 & $0.00 \%$ \\
\hline$(2.75 ; 3]$ & 4 & $0.60 \%$ & 0 & $0.00 \%$ \\
\hline$(3 ; 3.25]$ & 2 & $0.30 \%$ & 0 & $0.00 \%$ \\
\hline$(3.25 ; 3.5]$ & 2 & $0.30 \%$ & 1 & $0.15 \%$ \\
\hline$(3.5 ; 3.75]$ & 2 & $0.30 \%$ & 0 & $0.00 \%$ \\
\hline$(3.75 ; 4]$ & 1 & $0.15 \%$ & 0 & $0.00 \%$ \\
\hline$(4 ; 4.25]$ & 0 & $0.00 \%$ & 0 & $0.00 \%$ \\
\hline$(4.25 ; 4.5]$ & 1 & $0.15 \%$ & 0 & $0.00 \%$ \\
\hline
\end{tabular}

malignant infiltration of tumor is much less varied in comparison to its true range (Table 1). The value of [Marg EPM] however is to some extent subjective. Most likely, in the evaluation of individual surgeons and in accordance to their experience, it may vary. Despite this, aiming to objectify the characteristics of MMS in relation to EPM, we undertook to determine this value at the clearly defined level.

The index of radicality of tumor removal [IRTR] [Marg max MMS] / [Marg EPM] ranged from 33,33\% to $444 \%$, on average $106.2 \%$. This indicates that the lateral excision was performed in MMS on average $6.2 \%$ more radically compared to the standard EPM (Table 2, Figure 4). Nevertheless, the difference between the individual components of this value [Marg max MMS] and [Marg EPM] was not statistically singificant $(\mathrm{p}=0,157)$. In 84 cases $(12.57 \%)$ the width of the furthest excision in the CMM was the same as the width of the EPM (red line in Figure 4 at level of $100 \%$ ), corresponding to the cases in which surgical excision and MMS would be complete and radical (effective) in the same degree. In 345 cases (51.64\%) [IRTR] was lower than $100 \%$ (red line in graph 2 below 100\%), corresponding to the cases in which surgical excision would be admittedly effective, but nevertheless made with a certain excess of healthy tissue (defined by a specific percentage - differing from $100 \%)$. In 237 cases (35.47\%) - [IRTR] exceeded 100\% (red line in graph 2 above $100 \%$ ), and that applied to the tumors, in which employing the classic surgical excision would leave residual tumor tissues. In a small subset of tumors with the most aggressive malignant infiltration - completely impossible to predictsuch transgressions reached several hundred percent. Comparative studies on the width of the excision in the MMS and EPM have also been conducted by other authors. For instance Bumsted having treated 71 tumors of auricle, compared the designed standard excision margin with the margin employed in the MMS. According to him, the standard margin would be inefficient in about $20 \%$ of cases [10]. In our material, the frequency of transgressions of "classic" excision margins was greater $(31.1 \%)$, which indicates high average aggressiveness of tumors treated.

The index of the extent of post-operative defect [IED] [Marg mean MMS] / [Marg EPM] ranged in each case from 33,33\% to $344.41 \%$

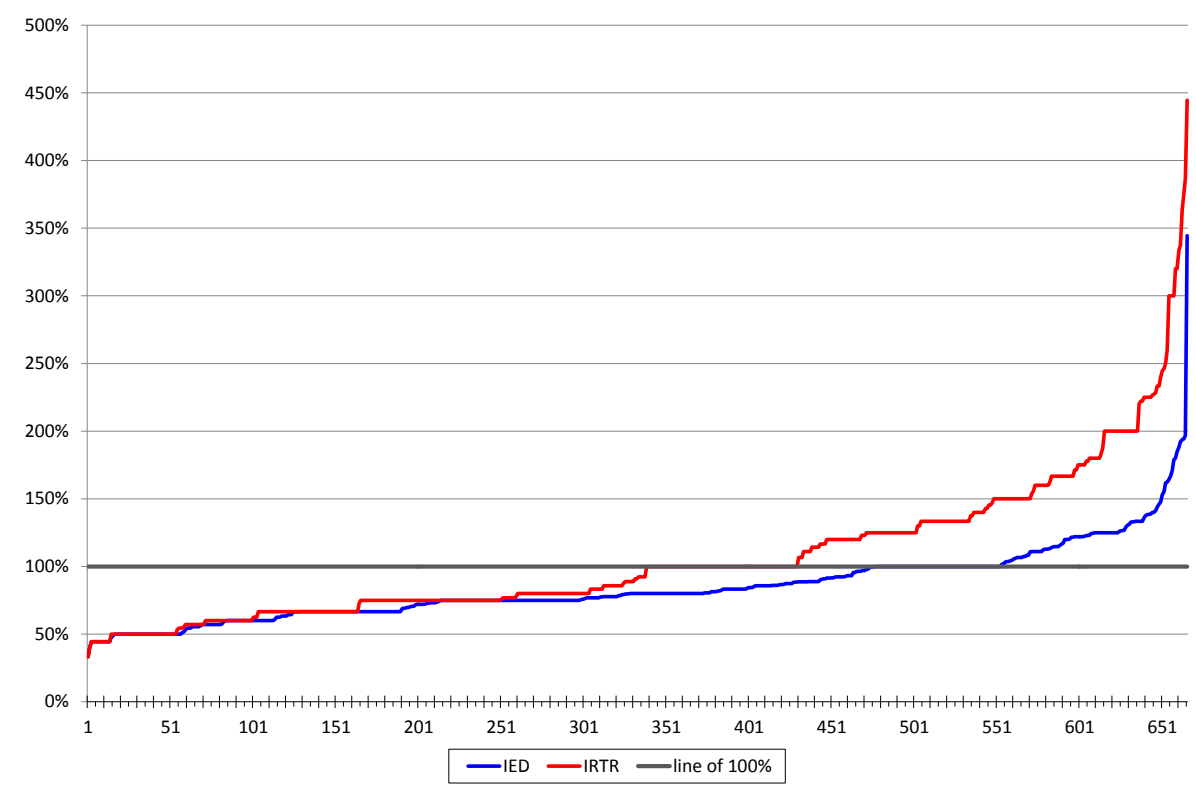

Figure 4. Index of radicality of tumor removal [IRTR] -red line, and index of the extent of the defect [IED] (blue line) calculated during treatment of 668 Basal Cell Cancers with Mohs Micrographic Surgery method. (X-axis - depicts number of patients, $\mathrm{Y}$-axis depicts values of given indexes). 
Bieniek A (2017) The new indexes comparing the radicality of tumor removal and the extent of post-operative defects after treatment of basal cell cancer by mean of Mohs Surgery and Classical Excision

(average of $84.93 \%$ ) (Table 2, Figure 4). This means that the average extensiveness of the defects after the treatment by MMS technique was $15.07 \%$ lower compared to the standard surgical resection EPM. Besides, the difference between the individual components of this value [Marg mean MMS] and [Marg EPM] was statistically singificant $(p=0,000)$. In 77 cases $(11.52 \%)$, the average width of the margin after excision with MMS was equal to the hypothetical standard excision width, which corresponded to the cases in which defects after treatment with both methods would exhibit the same size (blue line in graph 2 at level of $100 \%)$. In 475 cases (71.10\%), the mean width of the margin after excision with MMS was smaller than after the standard one (blue line in graph 2 below 100\%), what demonstrates cases in which the extent of the defect after excision with MMS was smaller than after EPM. Nevertheless, in 116 cases $(17.36 \%)$ - the mean width of the margin after excision with MMS was greater than after the EPM (blue line in graph 2 above 100\%). In many cases it was even several times greater, which corresponded to cases of most aggressive and unexpected tumor invasion. According to the data presented, in most cases tumors were removed with smaller mean width than routine recommendations would suggest. This usually leaded to minimized sacrifice of tissue, which assured good aesthetic result thanks to spontaneous healing or less extensive reconstructions. However, as stated above, not infrequent were also the situations in which the treatment was started with the intention of sparing tissue, whereas the final size of the defect was significantly greater than the hypothetical deficit after standard excision. Studies on the extent of surgical defects in MMS were also conducted by other researchers. Inkster, for example, by comparing the size of the defect after the MMS with the defects after standard excision, concluded that they were in $20 \%$ greater, in $37 \%$ - smaller, and the rest (about 43\%) was comparable in size [11]. In the material published by Bumsted and others, in approximately $80 \%$ of cases, hypothetical defects after the standard excision significantly exceeds the defect after MMS (an average of $180 \%$ in primary tumors and $27 \%$ in secondary tumors) [10]. Due to unique properties of MMS, in $14,97 \%$ of cases (126 cases), excision was both more radical [IRTR] $>100 \%$, and at the same time- more tissue sparing [IED] $<100 \%$.

To the authors' assessment the newly described indexes may serve as precise tools for comparison of MMS and the standard surgical excision, both in individual cases and in larger groups of patients. Standard excision with margins designed prior to the surgery, with subsequent histological examination by conventional histological technique with paraffin-based processing is in most countries regarded as the method of choice in the treatment of skin cancers. Due to the prevalence of this method, surgeons mostly do not deeply analyze its properties, believing that sacrifice of a significant amount of healthy tissue or considerable risk of treatment failure are inevitable. It appears that the presentation of detailed comparative studies about the character of tumor removal, and extensiveness of the defects (in EPM and MMS) convinces that individual tailoring of excision may be better than adherence to more or less universal "template". Yet, even in the world of fashion "pret a porter” cannot equal to „haute couture”.

\section{Conclusions}

During the 668 operations of BCC with use of MMS it was found, that:

The average index of radicality of tumor removal [IRTR] was 106,2, what indicated, that average radicality of tumor removal in MMS exceeded the radicality after a standard Excision with Predetermined Margins by $6.2 \%$ (not statistically significant; $\mathrm{p}=0,157$ ).

The average index of extent of the defect in MMS [IED] was $84,93 \%$, what indicated, that average extent of post-operative defects in MMS was smaller as compared to the standard surgical Excision with Predetermined Margins by 15,07\% (statistically significant; $\mathrm{p}=0,00$ ).

Because of frequent unpredictable direction and range of tumor infiltrations, the technique of MMS allowing for its appropriate excision, makes it possible to maintain a high radicality of tumor removal and optimize the size of the lesions, in comparison to the standard surgical Excision with Predetermined Margins.

\section{References}

1. Leffell DJ, Carucci JA (2001) Managment of Skin Cancer In: Principles and Practice of Oncology (ed): DeVita VT Jr, Hellmann S, Rosenberg SA. Cancer. Wilkins Lippicott Williams, Philadelphia pp: 1976-2002.

2. Brodland DG (1993) Surgical margins for nonmelanoma skin cancers. In: Surgical Dermatology, Advances in current practice. (Ed) Roenigk RK, Roenigk HK, Dunitz M, London pp: 139-144.

3. Breuninger H, Dietz K (1991) Prediction of subclinical tumor infiltration in basal cel carcinoma. J Dermatol Surg Oncol 17: 574-578. [Crossref]

4. Wolf DJ, Zitelli JA (1987) Surgical margins for basal cell carcinoma. Arch Dermatol 123: 340-344. [Crossref]

5. Sexton M, Jones DB, Maloney ME (1990) Histologic pattern analysis of basal cell carcinoma. Study of a series of 1039 consecutive neoplasms. J Am Acad Dermatol 23: 1118-1126. [Crossref]

6. Mosterd K, Krekels G, Nieman FHM, Ostertag JU, Essers BAB, et al. (2008) Surgica excision versus Mohs' micrographic surgery for primary and recurrent basal-cel carcinoma of the face; a prospective randomized controlled trial with 5-years follow up. Lancet Oncol 9: 1149-1156.

7. Breuninger H, Konz B, Burg G (2007) Microscopically Controlled Surgery in Malignant Dermatological Tumors. Dtsch Arztebl 104: A-427-432.

8. Malhotra R, Huilgol SC, Huynh NT, Selva D (2004) The Australian Mohs database, part II: periocular basal cell carcinoma outcome at 5-year follow-up. Ophthalmology 111: 631-636. [Crossref]

9. Batra RS, Kelley LC (2002) Predictors of extensive subclinical spread in nonmelanoma skin cancer treated with Mohs micrographic surgery. Arch Dermatol 138: 1043-1051. [Crossref]

10. Bumsted RM, Ceilley RI, Panje WR, Crumley RL (1981) Auricular malignant neoplasms. When is chemotherapy (Mohs' technique) necessary? Arch Otolaryngol 107: 721-724. [Crossref]

11. Inkster C, Ashworth J, Murdoch JR, Montgomery P, Telfer NR, et al. (1998) Oculoplastic reconstruction following Mohs surgery. Eye (Lond) 12: 214-218. [Crossref]

Copyright: (C2017 Bieniek A. This is an open-access article distributed under the terms of the Creative Commons Attribution License, which permits unrestricted use, distribution, and reproduction in any medium, provided the original author and source are credited. 